\title{
Imported malaria: key messages in an era of elimination
}

\author{
Authors: Tommy Rampling, ${ }^{A}$ Colin J Sutherland ${ }^{B}$ and Christopher JM Whitty ${ }^{C}$
}

Despite concerted efforts to eliminate malaria, it remains a major global cause of morbidity and mortality with over 200 million annual cases. Significant gains have been made, with the annual global malaria incidence and mortality halving over the past twenty years, using tools such as longlasting insecticide-treated bed nets and artemisinin-based therapies. Malaria is also a significant cause of life-threatening imported infection in the UK. It is vital for front line clinical staff involved in the assessment of acutely ill patients to be aware of the need for early diagnostic testing, malaria epidemiology, markers of severe infection and developments in antimalarial treatments to optimise patient management. The difference between a good and poor outcome is early diagnosis and treatment. Many of the challenges faced in the quest for global eradication, such as availability of appropriate diagnostic tests, and drug and insecticide resistance could also have future implications for imported malaria.

\section{Introduction}

Malaria, caused by Plasmodium parasites and transmitted by anopheline mosquitoes, remains a major cause of global morbidity and mortality with an estimated 219 million cases in 2017 and 435,000 deaths. ${ }^{1}$ Recent efforts to control and eventually eliminate malaria have reduced the disease burden in several countries, but it remains an important cause of imported infection in the UK, with 1,792 cases reported in 2017, causing six deaths. Plasmodium falciparum accounts for $81 \%$ of UK infections. ${ }^{2}$ During the 20th century malaria was eliminated from many temperate regions, including England, but remains endemic to 91 countries worldwide. ${ }^{1}$ Several species of parasite cause human malaria (P falciparum, Plasmodium vivax, Plasmodium ovale curtisi, Plasmodium ovale wallikeri and Plasmodium malariae), with the zoonotic Plasmodium knowlesi an important human pathogen in parts of southeast Asia.

\footnotetext{
Authors: A academic clinical fellow in infectious diseases and medical virology, Hospital for Tropical Diseases, London, UK; ${ }^{B}$ professor of parasitology, Department of Immunology and Infection, London School of Hygiene \& Tropical Medicine, London, UK; ${ }^{C}$ professor of public and international health and consultant infectious diseases physician, Hospital for Tropical Diseases, London, UK
}

\section{Key messages for UK clinicians}

Updated UK malaria treatment guidelines were published in 2016. ${ }^{3}$ Here we highlight key messages and recent changes.

\section{Clinical presentation}

The incubation period and clinical presentation of malaria are highly variable and non-specific, and missed or late diagnosis is life-threatening. Symptoms begin during the blood stage of infection (Fig 1). Malaria should be considered in any patient presenting with fever or history of fever up to 1 year after returning from the tropics, or sometimes longer especially if $P$ vivax infection is a possibility. Common symptoms of uncomplicated malaria include fevers, chills, muscle and joint aches and headache. The key to diagnosis is prompt laboratory malaria testing. It is not possible to diagnose malaria based on clinical features alone. Severe malaria has a clearly defined set of clinical and laboratory criteria which must be systematically considered (Table 1); if patients have any of these they will need parenteral treatment. Elderly patients, and those who are pregnant are at particular risk of complications from malaria. ${ }^{4}$

\section{Key points}

Malaria must be considered in all patients with history of fever who visited malaria endemic areas within the preceding year

Blood film microscopy remains the gold standard for malaria diagnosis, with rapid diagnostic tests serving as useful adjuncts

Intravenous artesunate is the drug of choice for patients with severe falciparum malaria; oral artemisinin-based combinations are recommended for uncomplicated malaria

Malaria elimination is being pursued in several countries with the eventual aim of global eradication, but despite successes it remains common especially in Africa

Malaria science is advancing rapidly including a falciparum malaria vaccine and a new drug for vivax malaria relapse being licensed in the last two years

KEYWORDS: Malaria, imported infection, malaria elimination 


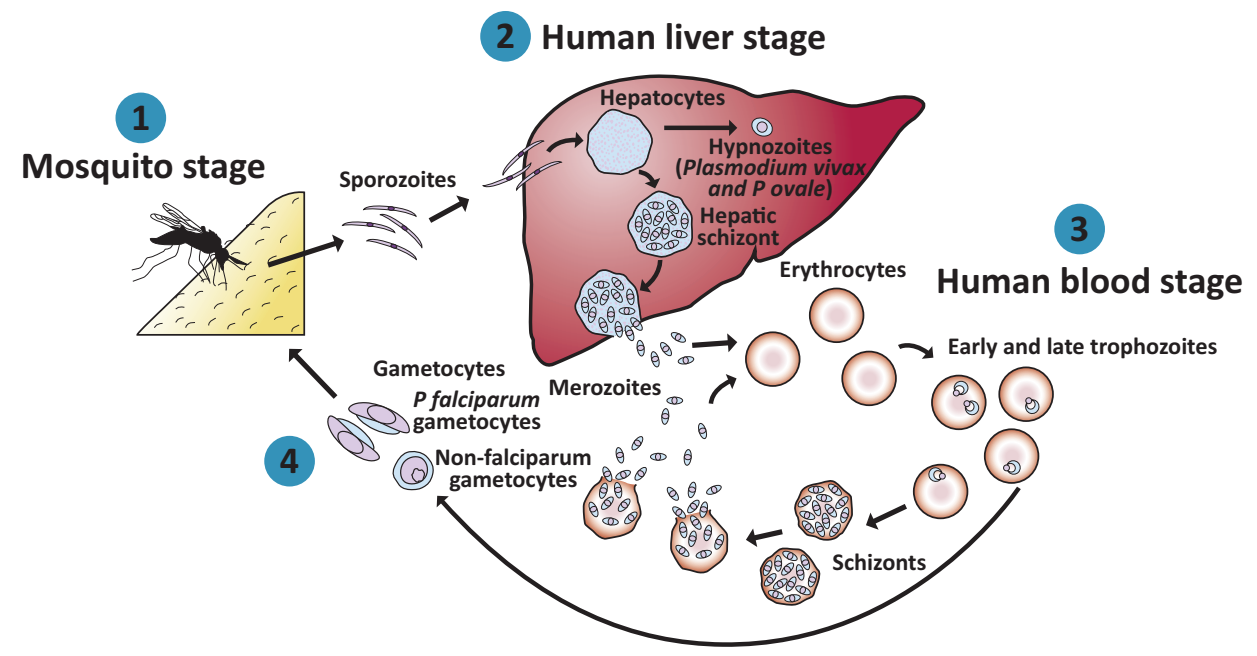

Fig 1. (a) The malaria parasite life cycle in humans begins when a malaria-infected female Anopheles mosquito takes a blood meal. Sporozoites in the mosquito salivary glands are inoculated into the human host. (b) The sporozoites travel via the blood to the liver in a matter of minutes, where they infect hepatocytes and mature into schizonts over days to weeks. This stage is asymptomatic. In Plasmodium vivax and Plasmodium ovale infection, a dormant stage (hypnozoites) can persist in the liver and cause relapses by invading the bloodstream weeks or even years later. Mature hepatic schizonts rupture and release merozoites which infect erythrocytes in the peripheral blood stream. (c) This marks the start of the blood stage of infection, during which the clinical manifestations of malaria occur. The parasites mature within the erythrocytes into ring stage trophozoites and then into schizonts, which rupture releasing more merozoites and allowing the blood stage cycle to begin again. (d) Some parasites differentiate into sexual erythrocytic stages (gametocytes) which are ingested by an Anopheles mosquito during a blood meal. The gametocytes mature in the mosquito through the sporogonic cycle, ultimately resulting in infective sporozoites in the salivary glands in preparation for inoculation into a new human host.

\section{Table 1. Major clinical and laboratory features of severe malaria}

Major features of severe or complicated falciparum malaria in adults.

Cerebral involvement: impaired consciousness, seizures and neurological signs

Pulmonary oedema and acute respiratory distress syndrome (ARDS)

Renal impairment (oliguria $<0.4 \mathrm{~mL} / \mathrm{kg}$ bodyweight per hour or creatinine $>265 \mathrm{mmol} / \mathrm{L}$ )

Metabolic and lactic acidosis $(\mathrm{pH}<7.3)$

Hypoglycemia $(<2.2 \mathrm{mmol} / \mathrm{L})$

Anaemia (haemoglobin $\leq 80 \mathrm{~g} / \mathrm{L}$ )

Spontaneous bleeding/disseminated intravascular coagulation

Hypovolaemia/shock (blood pressure $<90 / 60 \mathrm{mmHg}$ )

Haemoglobinuria (without glucose-6-phosphate dehydrogenase deficiency)

Parasitaemia $>10 \%$ (In the UK a parastitaemia of $>2 \%$ or $\leq 2 \%$ with schizonts present indicates potentially severe malaria, and is an indication for parenteral treatment)

Malaria can be more severe in pregnancy. Early consultation with a specialist unit should be made, and parenteral therapy should be considered.

\section{Diagnosis}

Examination of blood films remains the gold standard for malaria diagnosis. Microscopy confirms or excludes malaria, identifies malaria species, and determines the density of parasitaemia. Rapid diagnostic tests (RDTs) are an additional investigation and detect the presence of malaria antigens in a 'dipstick' format. ${ }^{5}$ RDTs are a useful adjunct, but not a replacement for blood film microscopy. Once malaria is diagnosed, treatment should start without delay.

Malaria is a notifiable disease in the UK. Blood films should be sent for confirmation to the Malaria Reference Laboratory in England and Wales, and the Scottish Parasite and Diagnostic Reference Laboratory in Scotland.

\section{Artemisinins}

Artemisinin derivative drugs are central to the management of malaria. ${ }^{6}$ Parenteral artesunate should be first line treatment in all patients diagnosed with severe malaria including children and all trimesters of pregnancy. Artesunate has been shown to reduce mortality compared to quinine in two large trials (SEAQUAMAT in south east Asia and AQUAMAT in Africa). ${ }^{7,8}$ Artesunate does not hold a UK license, but is available in many UK infectious diseases units and can be urgently supplied from specialist tropical medicine centres in London and Liverpool (contact details below). Malaria is life-threatening, and can progress rapidly, so if artesunate is not immediately available, treatment of severe malaria with intravenous (IV) quinine should not be delayed whilst artesunate is being obtained. If you are not experienced in managing severe malaria we recommend getting advice from a specialist centre for the 
complications including cerebral malaria, acute lung injury and renal failure, but in all situations prompt administration of high dose IV artesunate (or quinine if not available) is the most important intervention.

For non-severe malaria, oral artemisinin combination therapies (ACTs) are the treatment of choice. Two ACTs are licensed for use in the UK, artemether-lumefantrine $\left(\right.$ Riamet $\left.^{\circledR}\right)$ and dihydroartemesinin-piperaquine (Eurartesim $\left.{ }^{\circledR}\right)$; the former more commonly stocked by UK pharmacies.

\section{Non-falciparum malaria}

Non-falciparum malaria (vivax, ovale, malariae) can be treated with $\mathrm{ACT}$ or, if $\mathrm{ACT}$ is unavailable, with chloroquine. These infections are usually treated with oral drugs as outpatients, but if signs of severity or vomiting are present, admission for parental treatment is appropriate. Deaths do occur, especially in the elderly. ${ }^{9}$ In $P$ vivax and $P$ ovale spp malaria it is important to ensure eradication of hypnozoites in the liver to prevent relapse of infection. Primaquine is the current hypnozoitocidal drug, with 14 days of follow on treatment after the schizonticide recommended to prevent relapse. Patients may fail to complete the full 14 -day primaquine course. Primaquine treatment is complicated by haemolysis risk in patients with glucose-6-phosphate dehydrogenase (G6PD) deficiency which can be severe, and G6PD screening is required before primaquine can be given. A new drug, tafenoquine, has been given a license for vivax relapse by the US Food and Drug Administration in 2018. Tafenoquine is a single dose radical cure but has the same risks with G6PD deficiency as primaquine, so may increase adherence but does not reduce the safety risk.

\section{The global outlook: new directions}

\section{Malaria eradication and its implications for imported infection}

Significant progress towards local elimination of malaria has been made over the past 20 years with the aim of eventual global eradication. While control has improved substantially in some countries the slowest progress is in some of the highest transmission settings in sub-Saharan Africa and significant numbers of imported cases can be expected from Africa for the foreseeable future. ${ }^{1}$

The primary target for malaria elimination is $P$ falciparum. As transmission falls within a population there may be an overall decrease in the prevalence of partial immunity to falciparum malaria, and a consequent increase in the risk of severe disease in individuals who are infected. Assuming adults from Africa with imported malaria are semi-immune is increasingly unlikely to be a safe clinical approach. Hypnozoite-mediated relapse of vivax malaria is difficult to prevent, so an epidemiological shift towards a higher proportion of $P$ vivax infections can occur as $P$ falciparum infections become less prevalent. This is already apparent in parts of Asia and Latin America.

\section{Vector control}

The biggest impact on malaria incidence in high transmission settings has probably come from long-lasting insecticide treated nets (LLINs). The widespread distribution and increasing acceptance of LLINs has had a dramatic influence on malaria morbidity and mortality, but increasingly prevalent pyrethroid resistance in Anopheles mosquitoes is a potential threat to continued success which is difficult to combat. ${ }^{10}$ There is a risk that protection of travellers under LLINs will decrease.

\section{Drug resistance}

Like other micro-organisms, Plasmodium is capable of developing resistance under selective drug pressure. In the 1990s, the global spread of resistance to chloroquine and sulfadoxinepyrimethamine lead to increased morbidity and mortality, particularly in African children. ${ }^{11}$ The emergence and spread of artemisinin resistance in south east Asia over the past decade is now a major cause for concern and a serious potential threat to global health, given the reliance on artesunate for severe disease and ACTs for non-severe malaria. Artemisinin resistance causes delayed parasite clearance and post treatment gametocytaemia, suggesting increased potential for onward transmission. ${ }^{12}$ At present, proven artemsinin resistance is confined to Asia. The spread of artemisinin resistance into areas of higher endemicity, particularly certain areas in sub-Saharan Africa, would represent a major setback for malaria control. In the UK, there is limited but emerging evidence for treatment failure of non-severe imported malaria with artemether-lumefantrine. ${ }^{13}$ We need to remain vigilant for signs of reduced artemether-lumefantrine efficacy among imported cases of malaria. A shift to the more widespread use of alternative ACTs such as dihydroartemisinin-piperaquine in the future may be required.

\section{Diagnostic challenges in elimination and imported malaria}

Case finding is key to both a successful elimination programme and to diagnosing malaria in low-endemic settings, including imported malaria to non-endemic countries. Specialist parasitology laboratories have a very high sensitivity for clinical malaria, but this is not always shared by routine laboratories. We need better tests for identifying low parasite density infections (common in imported malaria), asymptomatic patients at risk of relapse with $P$ vivax and $P$ ovale spp, methods to identify pockets of high transmission where overall malaria incidence is falling and means to identify the last few cases at the end of an elimination campaign. These challenges would all benefit from more sensitive point of care tests. ${ }^{14} \mathrm{~A}$ concern identified over recent years is the emergence of strains of $P$ falciparum in the Americas and Africa with deletions in the PfHRP2 gene leading to false negative RDTs. ${ }^{15}$ It is not yet clear how widespread this is, but in malaria imported from some countries the proportion may be high enough to limit the usefulness of HRP2-only RDTs if used as the only diagnostic test. This is a persuasive reason to maintain malaria microscopy.

\section{Vaccine development}

An effective malaria vaccine would be transformational but has proved difficult to achieve. Many immunogens have been tried including whole irradiated parasites, viral vectors encoding malaria peptides, and protein subunits. ${ }^{16}$ The protein-in-adjuvant RTS,S/AS01 vaccine is the most advanced candidate, has been licensed for use in children in endemic countries and has been shown to elicit modest and relatively short-lived efficacy in phase 3 evaluation. ${ }^{17}$ A more substantial efficacy was observed in older children (36.3\% in 5 to 17 -month-olds). Whilst it may play an important role in control in some endemic settings, the current 
vaccine is unlikely to shift the epidemiology in most countries of relevance to travellers or to be effective as a travellers' vaccine against malaria.

\section{Conclusions}

Malaria remains an important cause of life-threatening imported infection in the UK; prompt diagnosis with microscopy and treatment are essential. Recent scientific developments including new drugs, diagnostics, a vaccine and initiatives for local elimination of malaria are important for control but will have limited impact on imported malaria in the next few years.

\section{Useful contacts}

Malaria Reference Laboratory: www.gov.uk/government/publications/ malaria-reference-laboratory-mrl-user-handbook.

Scottish Parasite and Diagnostic Reference Laboratory: www.nhsggc org.uk/about-us/professional-support-sites/scottish-microbiologyreference-laboratories/scottish-parasite-diagnostic-referencelaboratory.

Hospital for Tropical Diseases, UCLH, London: 02034567890.

Tropical and Infectious Disease Unit, Royal Liverpool University Hospital, Liverpool: 01517062000.

\section{References}

1 World Health Organization. World Malaria Report 2018. WHO, 2018.

2 Public Health England. Malaria imported into the United Kingdom: 2017. Public Health England, 2018.

3 Lalloo DG, Shingadia D, Bell D] et al. UK malaria treatment guidelines 2016. J infect 2016:72: 635-49.

4 Checkley AM, Smith A, Smith V et al. Risk factors for mortality from imported falciparum malaria in the United Kingdom over 20 years: an observational study. BMJ 2012;344:e2116.

5 Chiodini PL. Malaria diagnostics: now and the future. Parasitology 2014:141:1873-9.

6 Ashley EA, Pyae Phyo A, Woodrow C]. Malaria. Lancet 2018:391:1608-21.

7 Dondorp A, Nosten F, Stepniewska K et al. Artesunate versus quinine for treatment of severe falciparum malaria: a randomised trial. Lancet 2005;366:717-25.
8 Dondorp AM, Fanello CI, Hendriksen IC et al. Artesunate versus quinine in the treatment of severe falciparum malaria in African children (AQUAMAT): an open-label, randomised trial. Lancet 2010;376:1647-57.

9 Broderick C, Nadjm B, Smith V et al. Clinical, geographical, and temporal risk factors associated with presentation and outcome of vivax malaria imported into the United Kingdom over 27 years: observational study. BMJ 2015;350:h1703.

10 Protopopoff N, Mosha JF, Lukole E et al. Effectiveness of a longlasting piperonyl butoxide-treated insecticidal net and indoor residual spray interventions, separately and together, against malaria transmitted by pyrethroid-resistant mosquitoes: a cluster, randomised controlled, two-by-two factorial design trial. Lancet 2018;391:1577-88.

11 Snow RW, Trape JF, Marsh K. The past, present and future of childhood malaria mortality in Africa. Trends in parasitology 2001:17:593-7.

12 Ashley EA, Dhorda M, Fairhurst RM et al. Spread of artemisinin resistance in Plasmodium falciparum malaria. N Engl J Med 2014:371:411-23.

13 Sutherland CJ, Lansdell P, Sanders M et al. pfk13-independent treatment failure in four imported cases of Plasmodium falciparum malaria treated with artemether-lumefantrine in the United Kingdom. Antimicrob Agents Chemother 2017;61:e02382-16.

14 Tanner M, Greenwood B, Whitty C] et al. Malaria eradication and elimination: views on how to translate a vision into reality. BMC medicine 2015;13:167.

15 Gamboa D, Ho MF, Bendezu ] et al. A large proportion of $P$ falciparum isolates in the Amazon region of Peru lack pfhrp2 and pfhrp3: implications for malaria rapid diagnostic tests. PLoS One 2010;5:e8091.

16 Ouattara A, Laurens MB. Vaccines against malaria. Clin Infect Dis 2015;60:930-6.

17 RTS, S Clinical Trials Partnership. Efficacy and safety of RTS,S/AS01 malaria vaccine with or without a booster dose in infants and children in Africa: final results of a phase 3, individually randomised, controlled trial. Lancet 2015;386:31-45.

18 World Health Organization. World Malaria Report 2016. WHO, 2016.

Address for correspondence: Dr Tommy Rampling, Hospital for Tropical Diseases, Mortimer Market Centre, Capper Street off Tottenham Court Road, London WC1E 6AU, UK.

Email: t.rampling@ucl.ac.uk 